\title{
Use of the blue cotton screen method with endoscopy to detect occult esophageal foreign bodies
}

\author{
Yan Xia, Fan Zhang, Hong Xu, Weiran Xu \\ Department of Gastroenterology, The First Hospital of Jilin University, Changchun, Jilin, China
}

Videosurgery Miniinv 2017; 12 (4): 428-436

DOI: https://doi.org/10.5114/wiitm.2017.72326

\begin{abstract}
More than 20,000 cases of upper gastrointestinal foreign bodies (FBs) have been reported in the last 5 years in China. Early detection and treatment is vital in these patients. Differential diagnosis of esophageal injury and occult esophageal foreign bodies is challenging, particularly in the case of non-radio-opaque foreign bodies. A diagnostic technique with high accuracy and low risk is needed for clinical practice. We describe successful use of the "blue cotton screen method" to detect esophageal foreign bodies in 2 patients. The advantages and disadvantages of various diagnostic modalities in the management of patients with foreign body ingestion are presented. This technique is safer and more effective than traditional methods for foreign body impaction in the esophageal cavity. It could be applied for screening and in the differential diagnosis of esophageal injury and FBs in the esophageal lumen.
\end{abstract}

Key words: occult esophageal foreign bodies, endoscope, diagnosis, blue cotton screen method.

\section{Introduction}

Foreign body (FB) ingestion, especially of animal bones, is a common problem encountered in the emergency room. The majority of upper gastrointestinal foreign bodies are stuck in the esophagus [1]. Ingested FBs may cause esophageal perforation, even complicated with perforation of the aorta or carotid artery, and thyroid gland embedment. The diagnosis of esophageal FB relies on the clinical manifestations together with endoscopic and radiography [2, 3]. However, diagnosis of occult esophageal foreign bodies is inherently challenging, particularly in the case of invisible foreign bodies under endoscopy. This may affect the choice of treatment. Sometimes, absence of proper tools for exploration, or incorrect application of endoscopic biopsy forceps, or other tools, can lead to serious complications. Few studies have evaluated the validity of diagnosis pattern and risk factors of diagnoses.
With the progresses in endoscopic technology, flexible and rigid endoscopic methods are the most common approaches to extract FBs impacted in the esophagus. In contrast to rigid endoscopy, flexible endoscopy can be performed under local anesthesia in an outpatient setting, which effectively shortens the recovery time and is more cost-effective. Moreover, the risk of iatrogenic perforation is much lower with flexible endoscopy [4], though the preference for esophageal FB extraction is still controversial. Flexible endoscopy shows higher detection value before extraction. However, the diagnostic value of flexible endoscopy is still limited in some difficult cases. Thus, use of an assistant device with the flexible endoscope may improve the diagnostic efficacy in the evaluation of the characteristics of the FB and related complications. Diagnostic accuracy is essential for precise treatment, but there is still a lack of efficient assistant devices for assessment of esopha-

\section{Address for correspondence}

Hong Xu, Department of Gastroenterology, The First Hospital of Jilin University, Changchun, Jilin, China, phone: +86 043188782821 ,

e-mail: 37228481@qq.com 
geal FB [5], especially when the foreign bodies are too small to detect for emergency endoscopy. To overcome the difficulties of endoscopic management of FBs, we developed a new technology to detect FBs. Here, we report 2 cases of esophageal perforation caused by ingestion of occult fish bone, which was clinically unnoticed until its detection at onset of esophageal symptoms. We introduce a new device compatible with the flexible endoscope, which may help to minimize the risk of lethal complications of esophageal FBs. To the best of our knowledge, this is the first report to describe the use of flexible upper endoscopy with caps and a blue cotton tool to detect occult esophageal FBs.

The blue cotton screen method involves placement of a transparent cap at the tip of the endoscope. The cap is $10 \mathrm{~mm}$ long. A $20 \mathrm{~mm}$ diameter medical cotton ball stained with $2 \%$ methylene blue is placed on the cap. The biopsy forceps are taken out of the endoscopy working channel, then the blue cotton is clamped and the cotton pulled back about $20 \mathrm{~mm}$, and about $75 \%$ of the cotton is buried into the channel to avoid blocking the vision. After placing the endoscope into the esophagus, the cotton is grasped with biopsy forceps to reshape it about $20 \mathrm{~mm}$ long. Holding the endoscope, the biopsy forceps are rotated slowly in close contact with the esophageal mucosa. In the event of contact between the cotton and the $\mathrm{FB}$, the cotton yarn can be pulled up by the $\mathrm{FB}$, or part of the cotton would hang on the esophageal FB, which renders the foreign body easy to detect.

\section{Case report}

\section{Case 1}

A 47-year-old man presented at the emergency room (ER) with difficulty in breathing and persistent retrosternal pain which started after intake of a meal $2 \mathrm{~h}$ before. An ECG examination at a local clinic, half an hour after symptoms onset, showed ST segment elevation of $0.2-0.3 \mathrm{mV}$ in leads I, II, AVF, and v4-v6. The patient had no history of cardiovascular disease, smoking, or alcohol abuse. His vital signs were stable; physical examination was normal, except for percussion pain behind the sternum. Laboratory tests showed elevated white blood cells (WBC) $14.74 \times 10^{\%} /$; neutrophil percentage (NE \%) 0.81; high sensitivity C-reactive protein $18.8 \mathrm{mg} / \mathrm{l}$; and $\mathrm{cTnTI}<0.01 \mu \mathrm{g} / \mathrm{l}$. The patient was admitted to the cardiovascular department with a diagnosis of acute coronary syndrome/myocarditis. The thoracic computed tomography (CT) scan showed a sharp needle-like high-density shadow projecting out of the middle segment of the esophagus pointing towards the left atrium. No abnormal local changes were observed in the esophagus or the adjoining structures with the exception of mild pericardial effusion. The patient recalled a history of swallowing a fish bone during a meal about 2 days prior to admission. Endoscopy was performed to assess the possibility of removal of the foreign body. However, the foreign body could not be located. In the esophageal mucosa there was found only a mild mottled erosion at $33 \mathrm{~cm}$ from the incisors.

The patient was referred for surgery with thoracoscopy, but the FB could not be found. However, using the blue cotton screen technique (Photo 1), cotton fiber was seen hanging at the site of the erosion, which indicated the location of the FB. The patient was managed by both thoracoscopy and endoscopy. The foreign body was extracted through the endoscope. The patient was discharged without any complications after 15 days.

\section{Case 2}

A 52-year-old man presented at the emergency room with complaints of retrosternal discomfort during swallowing for 7 days, and a history of tarry stool and hematemesis were found in the last $12 \mathrm{~h}$. He gave a history of swallowing a bone by mistake. The patient felt dizzy and nauseous. There was no other digestive tract symptom or systemic symptom such as fever. He had a past history of alcoholism, but no history of chronic liver disease or peptic ulceration. His blood pressure was $105 / 70 \mathrm{~mm} \mathrm{Hg}$, pulse rate was 105 beats per minute and respiratory rate was 22 breaths per minute. He was administered $20 \mathrm{ml}$ of $5 \%$ glucose and omeprazole $80 \mathrm{ml}$ static push, $100 \mathrm{ml}$ of $5 \%$ glucose and $40 \mathrm{mg}$ omeprazole continuous infusion, $0.9 \%$ sodium chloride $500 \mathrm{ml}$ plus $6 \mathrm{U}$ insulin. No abnormality was detected on physical examination, except diaphoresis and mild pallor. Haematological investigations revealed mildly elevated white cell count $11.8 \times 10^{9} /$, with neutrophilia of $10.34 \times 10^{9} / \mathrm{l}$. His hemoglobin was $97 \mathrm{~g} / \mathrm{l}$. Standing chest and plain abdominal radiographs were normal. Computed tomography scan of the mediastinum showed indistinct esophagus middle, slightly higher density of the esophageal wall, the 

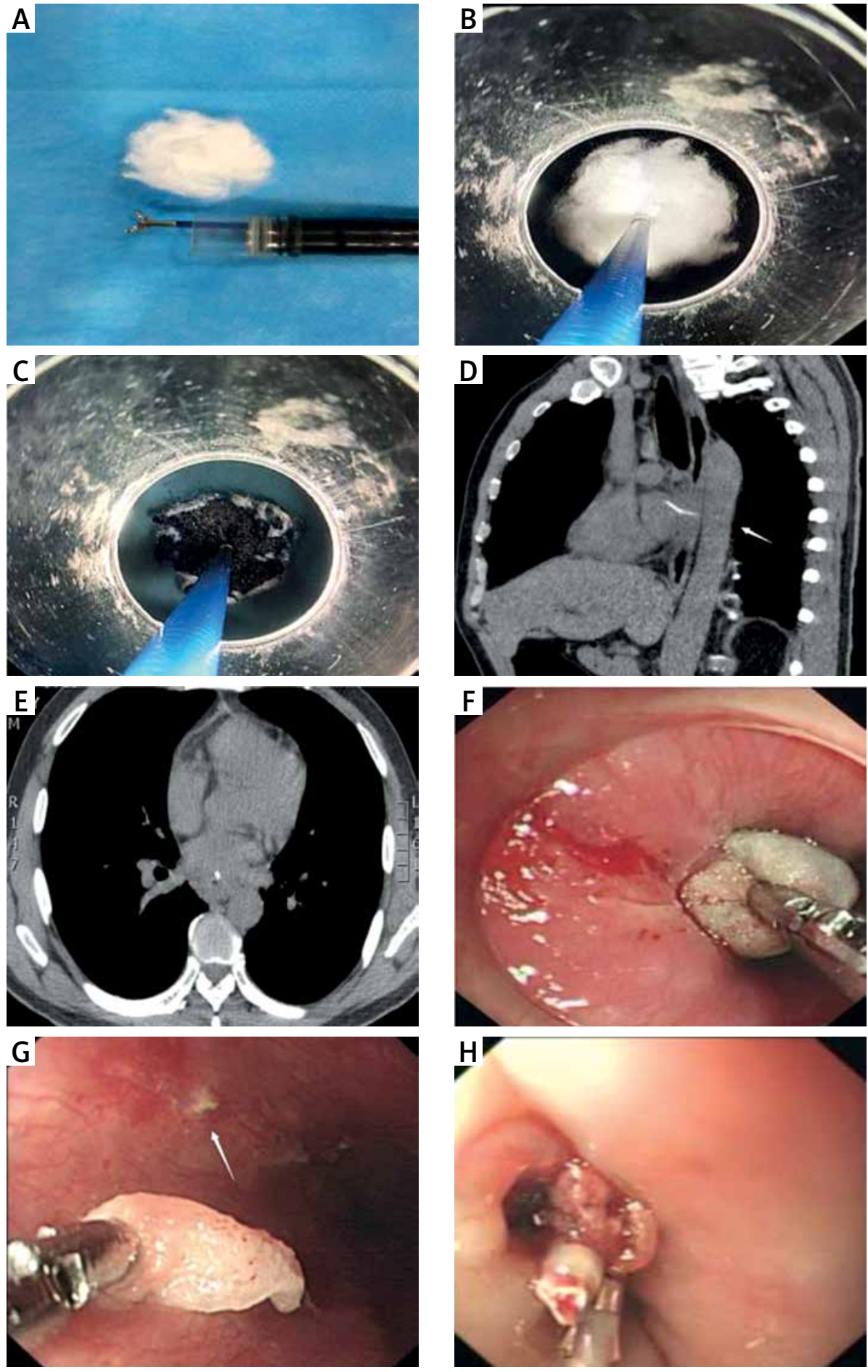

Photo 1. A - Blue cotton screen modality includes cotton, cap, biopsy forceps, and superfine flexible endoscopy. B, C - Endoscopic image showed the cotton before and after dye. D, E - Thoracic CT scan showed a sharp needle-like high-density shadow projecting out of the middle segment of the esophagus pointing towards the left atrium. Arrow shows esophageal foreign body. F-Endoscopic inspection found esophageal mucosa defect, no active bleeding. G - Application of blue cotton screen technology; arrow shows cotton attachment on the esophageal mucosa defect, suspected esophageal foreign body. $\mathbf{H}-$ After removal of the foreign body and closure of the mucosa by clips under the endoscope 
surrounding fat tissue had disappeared, part of the descending aorta showed aorto-esophageal fistula, and there was an esophageal foreign body. Emergency endoscopy revealed no upper esophageal FB. There was a fresh red thrombus at $26 \mathrm{~cm}$, with no active bleeding. The foreign body was not visible. Foreign body was also not found during surgery with thoracoscopy. Then a second endoscopy examination was performed, and the blue cotton method was conducted after water washing, then a small piece of cotton was located at $26 \mathrm{~cm}$, and a tiny part of a fish bone was found under the cotton (Photo 2). The foreign body was extracted by the endoscope. The patient was discharged without any complications after 23 days.

\section{Discussion}

In clinical practice, it is difficult to diagnose occult esophageal foreign bodies, particularly when no foreign bodies are detected by endoscopy. As shown in Table I, FBs may be missed on endoscopy, although supplemental use of other devices may overcome this limitation. The clinical manifestations are not often in accordance with the site of upper gastrointestinal FB congestion. Patients with esophageal erosion and those with esophageal FB often have similar early symptoms. Non-specific symptoms may lead to misdiagnosis, such as pneumonia, ulcer, myocardial infarction, and spontaneous pneumothorax [6]. Diagnosis and removal of FB impacted in the esophagus can sometimes be very challenging. Prompt diagnosis and subsequent treatment for esophageal FB are essential to minimize complications.

\section{Radiographic imaging}

Radiography of the chest and neck is clinically useful because of its ready availability and noninvasive nature. X-rays are typically useful for detection of radiopaque objects, emphysema in the neck or mediastinum, and pleural effusion. The European Society of Gastrointestinal Endoscopy (ESGE) does not recommend radiological evaluation for patients with no bone or food bolus impaction without complications. We recommend plain film, both anterioposterior and lateral, to evaluate the presence, location, size, configuration, and number of ingested FBs in cases with suspected objects [6].

However, non-radio-opaque esophageal FBs may not be detected by radiography when the patient ex- hibits non-specific respiratory symptoms. Numerous objects, such as food boluses, small bones, wood, plastic, glass, and thin metal, are often not detected on radiography. The sensitivity of X-ray for detection of ingested bones is only about $25 \%$ to $57 \%$, while it is much lower in detecting more proximal upper esophageal impactions to incisors. Varied densities, sizes, and locations of the bone may also increase the false negative results. Furthermore, the way that the FB is impacted in the esophagus cannot be confirmed on chest radiography, as these FBs may be indistinguishable from adjacent tissues [7]. Although chest radiograph is recommended in the majority of patients with esophageal perforation, it may be normal at the beginning. At least $1 \mathrm{~h}$ is needed for mediastinal emphysema to present discernible radiographic evidence of injury, while a much longer time is required for pleural effusion and mediastinal widening to be detectable.

Fluoroscopy with water-soluble contrast agent has been performed in cases of non-radiopaque FB ingestion, or in patients suffering from esophageal cancer or esophageal stenosis. Extraluminal extravasation from the esophagus can confirm the existence of perforation, whereas a filling defect may indicate larger FB impaction. Esophagography using water-soluble contrast medium is the gold standard for perforation diagnosis. As the false negative rate is up to $60 \%$, esophagography is useless in the diagnosis of cervical perforation in spite of its pivotal role in thoracic perforation.

Barium meal examination is not the optimal choice for initial FB detection as it may interfere with the subsequent surgical or radiographic visual field. Thus, barium esophagography, in spite of being a sensitive test for esophageal perforation, should be performed only after negative fluoroscopy and in the absence of indications for surgery. In addition, the application of barium should be limited when there is suspected perforation, as extravasation may lead to soft tissue inflammation. In fear of aspiration and impairment of the endoscopic view, the ESGE does not recommend barium meal for esophageal FB [6].

\section{Computed tomography}

Computed tomography is indispensable to the diagnosis of esophageal FB and perforation. Computed tomography imaging provides rich information on the nature of the FB, its location, evidence of 

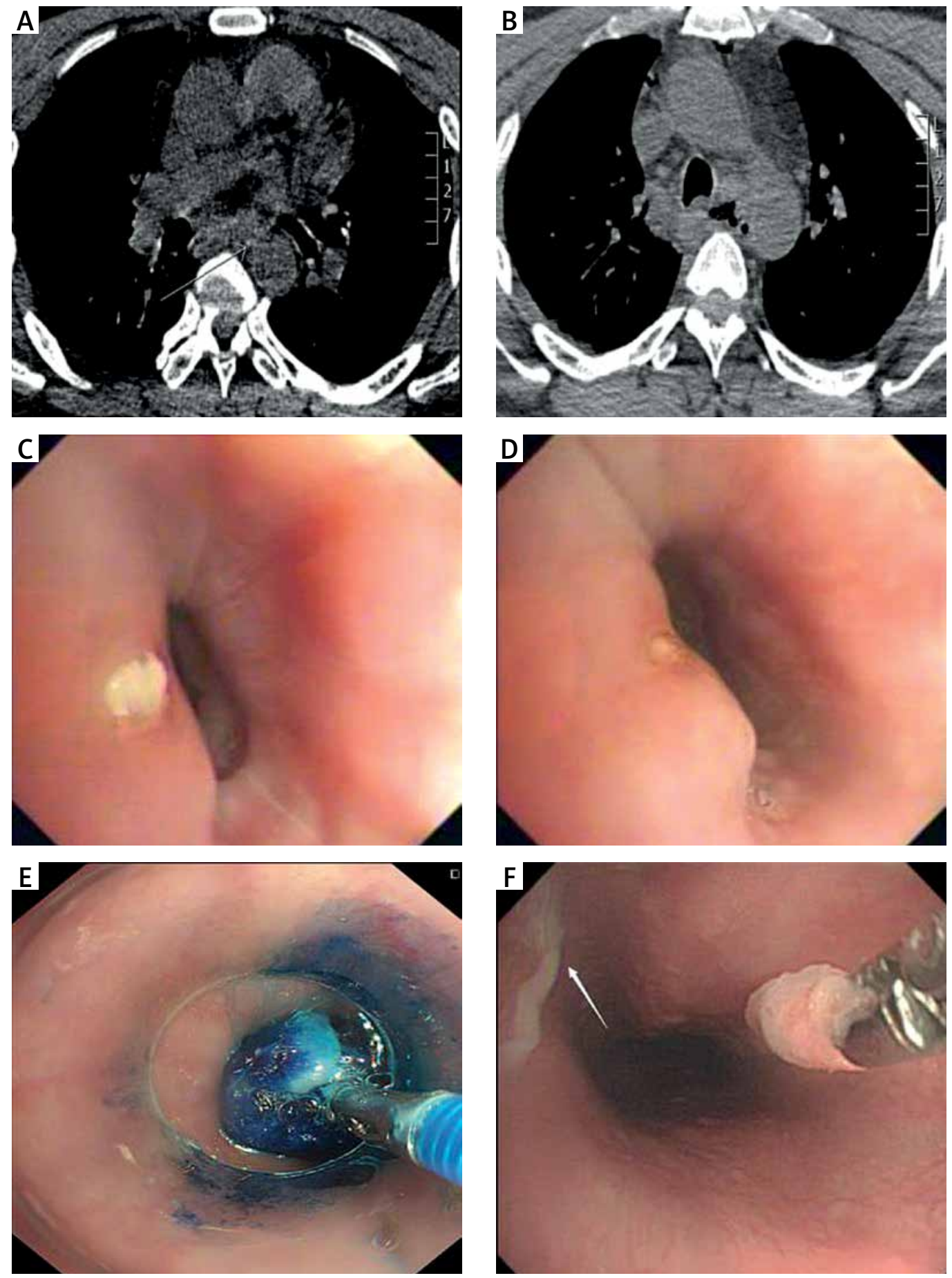

Photo 2. A, B - Computed tomography scan of the mediastinum showed indistinct esophagus middle, slightly higher density of esophageal wall, the surrounding the fat gap has disappeared, part of the descending aorta shows ill location, arrow shows aorto-esophageal fistula and esophageal foreign body. C - Emergency endoscopy revealed a clean upper esophagus from the incisors. There was a fresh red thrombus at $26 \mathrm{~cm}$, with no active bleeding. The foreign body was not visible. D - After washing and screening with blue cotton, a small defect was observed. E - Use of blue cotton screen method to check the suspected foreign body at the defect. F - Arrow shows cotton attachment on the esophageal mucosa defect, suspected esophageal foreign body 
Table I. Studies using various techniques to detect foreign bodies

\begin{tabular}{|lcclcc|}
\hline Author, year & Study site & $N$ & Study type & Diagnosis type & Complication \\
\hline Radford, Wells, 1988 [13] & England & 1 & Case report & Surgery+, E- & Perforation \\
\hline Chung, 2003 [14] & Hong Kong & 1 & Case report & CT+, E- & Perforation \\
\hline Katsetos et al., 2003 [15] & United States & 1 & Case report & Autopsy+, E- & Dead \\
\hline De Lucas et al., 2004 [16] & Spain & 1 & Case report & CT+, E- & Perforation \\
\hline Ng, 2005 [17] & Hong Kong & 1 & Case report & Autopsy+, E- & Dead \\
\hline Metz et al., 2006 [18] & Netherlands & 1 & Case report & CT+, E- & Fistula \\
\hline Tsalis et al., 2009 [19] & Greece & 1 & Case series & CT+, E- & Abscess \\
\hline Sockeel et al., 2009 [20] & France & 2 & Case report & CT+, E- & Perforation \\
\hline Bhat et al., 2009 [21] & Qatar & 1 & Case series & CT+, E- & Inflammation \\
\hline Huang et al., 2010 [22] & Spain & 1 & Case report & CT+, E- & Perforation \\
\hline Chen et al., 2010 [23] & China & 3 & Case series & CT+, E- & Perforation \\
\hline Kerl et al., 2010 [24] & Germany & 1 & Case report & MR+, E- & Abscess \\
\hline Chen et al., 2011 [25] & China & 2 & Case series & CT-/+, E- & Fistula \\
\hline Sung et al., 2011 [26] & Korea & 4 & Retrospective & X+, CT+, E- & Perforation \\
\hline Peng et al., 2012 [27] & China & 3 & Retrospective & CT-, Surgery+, E- & Abscess \\
\hline Søreide et al., 2012 [28] & Norway & 2 & Case report & CT-, Surgery+, E- & Perforation \\
\hline Ko et al., 2013 [29] & Taiwan & 1 & Case report & CT+, E- & Perforation \\
\hline Tsukiyama et al., 2014 [30] & Japan & 1 & Case series & CT+, E- & Perforation \\
\hline
\end{tabular}

perforation, bleeding, early soft tissue emphysema, inflammation in the chest, as well as fluid collections $[7,8]$. Typically, a variety of abnormities including extraluminal gas collection, pneumomediastinum, esophageal wall thickening, esophago-pleural fistula, pleural effusions, para-esophageal abscess cavity, and air communication between the esophagus and mediastinum are readily detected on CT. Of these, extraluminal gas collection is most commonly found on $\mathrm{CT}$ when considering esophageal perforation. Moreover, a CT scan is particularly useful for treatment decision-making.

Computed tomography imaging increases the diagnostic accuracy and aids clinical decision-making in stable patients with atypical signs and symptoms owing to its high sensitivity and a low false-negative rate. Multi-detector row CT (MDCT) has a shorter scanning time and reduced contrast agent usage, and offers higher resolution combined with 3D reconstruction. Furthermore, thoracic CT is particularly valuable in cases of suspected esophageal FB with negative results, but in whom esophagography is contraindicated or in cases with atypical symptoms with a history of esophageal injury $[9,10]$. Enhanced MDCT can help determine the nature of fluid in the lesions and injury of aorta and esophagus [11]. Moreover, enhanced MDCT together with 3D reconstruction or MDCT angiography can be adopted in aorto-esophageal fistula (AEF) and esophageal FB.

Interestingly, a CT scan may show fish bone impaction in patients who exhibit no FB in the following endoscopy or surgery. If the FB is not detected or located accurately, there are chances of serious complications. The reasons for misdiagnosis may be as follows: (1) Sharp FB aspiration can cause esophageal bleeding and acute mucosal injury. The esophageal wall may exhibit slightly higher density on CT examination, which is often misdiagnosed as a small FB, such as poor calcification of a small fishbone or other medium-textured foreign material. (2) By the impact of swallowing, esophageal wall 
contraction, and breathing instability, some images may produce artifacts that are similar to the image of a foreign body. Such a situation is especially pronounced in multi-planar reconstruction (MPR) images, which seriously restrains the application of CT examination. (3) At present, some CT scans cannot give the precise location of a foreign body inserted into the esophageal wall at an early time, limited by low resolution.

\section{Esophageal endoscopy}

Endoscopy is most widely used for diagnosis and removal of esophageal FBs. Although endoscopy is not recommended as the primary diagnostic modality of choice, it allows visual examination of the FB and potential perforation. Rigid or flexible endoscopy is the current gold standard for confirmation of the FB and perforation. The guidelines recommend that a sharp object or disk battery located in the esophagus should be treated with urgent endoscopic intervention [12]. ESGE recommends the application of emergent therapeutic esophageal endoscopy for FBs inducing complete esophageal obstruction, sharp objects, magnets, or batteries in the esophagus [6].

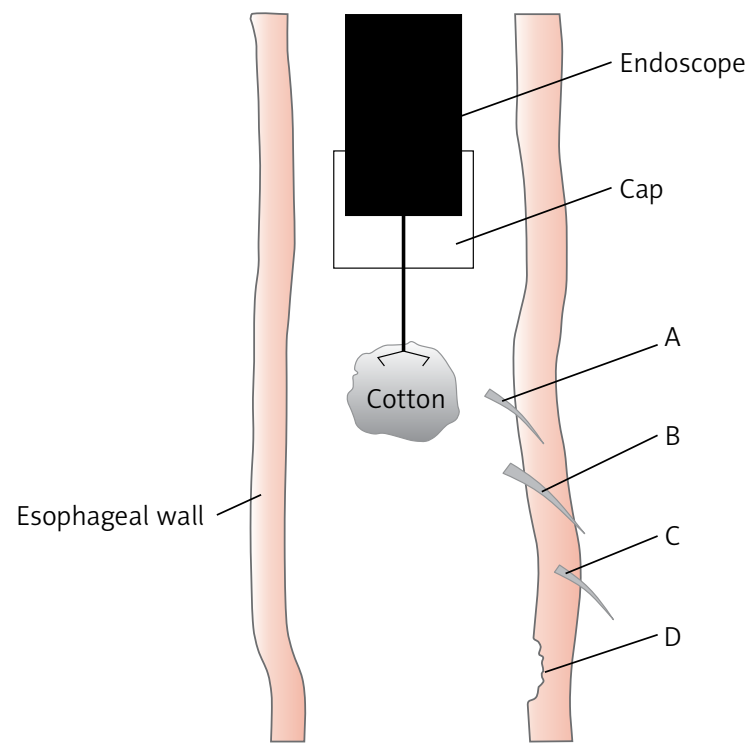

Figure 1. Schematic illustration of the blue cotton screen method. A - Foreign body impaction, some residues in esophagus. B - Transmural foreign body with small amount of residual in esophagus. C - Foreign body completely punctures the esophagus, invisible in esophagus. D - Foreign body scratching esophagus
Although endoscopy can visualize lesions in the lumen of the esophagus, it cannot detect FBs penetrating the esophageal wall or into the surrounding tissue. Small mucosal lesions may be easily missed on endoscopy. The application of endoscopy in suspected esophageal injury is limited, due to the risk of missing a perforation hidden in a mucosal fold and the risk of aggravating a small tear to perforation. Endoscopic examination may even cause iatrogenic injury when the FB is at the aortic arch level, as the FB may move outward and pierce the adjacent aortic wall. Endoscopic evaluation requires injected air; this will aggravate perforations.

\section{New technology}

Accurate examination is essential to guide treatment, but there is still a lack of efficient auxiliary examinations for esophageal FB ingestion [5]. Endoscopy is most urgently needed for locating tiny lesions in the esophagus. To overcome the above shortcomings of endoscopy, we developed a new technique to screen for FBs more efficiently. In the 2 presented cases, blue stained cotton helped identify the location of the lesion detected by CT and thus demonstrated the effectiveness of the blue cotton method. We refer to our original method as the "blue cotton screen method".

We suggest use of the blue cotton screen technology in the following conditions (Figure 1): (1) FB not embedded in the esophageal wall, and penetrating the esophageal wall. Under direct vision, we can identify the FB location, because the cotton can be pulled up by the FB, as the cotton will neither displace the FB nor cause injury to the esophageal wall. (2) FB embedded in as well as penetrating the esophageal wall. The routine endoscopic method is not allowed in this case, because of the need to inject gas to hold open the esophageal wall, which may further expand the esophageal perforation and lead to serious complications. With our blue cotton screen method, the cap supports the esophageal wall, allowing a better visual field. This method can be performed in some contraindicated cases, because we do not need to inject anything. The cotton can be made to contact the entire esophageal mucosa. During this process, the cotton fiber will pull up or hang any small mucosal projection caused by the FB, which helps to locate the FB. (3) FB completely penetrating the esophageal wall. In this case, 
if the thoracoscope cannot find the FB, we could use the blue cotton screen technology to check the esophageal wall with the thoracoscope. If the cotton contacts the FB, we may see the FB pull up the cotton silk, which may help to locate the foreign body. (4) FB scratching esophagus. Application of the blue cotton screen method in this case can allow evaluation of the wound and existence of the FB without aggravating the damage. It can facilitate differential diagnosis of esophageal injury and occult esophageal foreign bodies.

When the FB is found, it is necessary to inject gas to inflate the esophageal lumen. We recommend using carbon dioxide gas, as it is more readily absorbable in the human body than air. We suggest using sedative endoscopy for screening and treatment and avoiding sudden movement of the patient to avoid esophageal injury.

\section{Conclusions}

The "blue cotton screen method" is useful for detection of occult esophageal foreign bodies, such as in situations where no foreign bodies could be found using traditional endoscopy.

Application of the blue cotton screen method within $24 \mathrm{~h}$ by endoscopic examination is necessary if there is a high clinical suspicion of impacted foreign bodies in the esophagus.

\section{Acknowledgments}

We thank Medjaden Bioscience Limited for assisting in the preparation of this manuscript.

This work was supported by the Industrial Innovation Fund Project of Jilin Province (\#2016C052-3).

\section{Conflict of interest}

The authors declare no conflict of interest.

\section{References}

1. Lin J H, Fang J, Wang D, et al. Chinese expert consensus on the endoscopic management of foreign bodies in the upper gastrointestinal tract (2015, Shanghai, China). J Dig Dis 2016; 17: 65-78.

2. Ramareddy RS, Alladi A. Review of esophageal injuries and stenosis: lessons learn and current concepts of management. J Indian Assoc Pediatr Surg 2016; 21: 139-43.

3. Burgos A, Rabago L, Triana P. Western view of the management of gastroesophageal foreign bodies. World J Gastrointest Endosc 2016; 8: 378-84.
4. Yan XE, Zhou LY, Lin SR, et al. Therapeutic effect of esophageal foreign body extraction management: flexible versus rigid endoscopy in 216 adults of Beijing. Med Sci Monit 2014; 20: 2054-60.

5. Tseng CC, Hsiao TY, Hsu WC. Comparison of rigid and flexible endoscopy for removing esophageal foreign bodies in an emergency. J Formos Med Assoc 2016; 115: 639-44.

6. Birk M, Bauerfeind P, Deprez PH, et al. Removal of foreign bodies in the upper gastrointestinal tract in adults: European Society of Gastrointestinal Endoscopy (ESGE) Clinical Guideline. Endoscopy 2016; 48: 489-96.

7. Kim JP, Kwon OJ, Shim HS, et al. Analysis of clinical feature and management of fish bone ingestion of upper gastrointestinal tract. Clin Exp Otorhinolaryngol 2015; 8: 261-7.

8. Woo SH, Kim KH. Proposal for methods of diagnosis of fish bone foreign body in the esophagus. Laryngoscope 2015; 125: 2472-5.

9. Wei Y, Chen L, Wang Y, et al. Proposed management protocol for ingested esophageal foreign body and aortoesophageal fistula: a single-center experience. Int J Clin Exp Med 2015; 8: 607-15.

10. Park S, Choi DS, Shin HS, et al. Fish bone foreign bodies in the pharynx and upper esophagus: evaluation with 64-slice MDCT. Acta Radiol 2014; 55: 8-13.

11. Karmazanovsky GG, Buryakina SA, Kondratiev EV, et al. Value of two-phase dynamic multidetector computed tomography in differential diagnosis of post-inflammatory strictures from esophageal cancer. World J Gastroenterol 2015; 21: 8878-87.

12. Eisen GM, Baron TH, Dominitz JA, et al. Guideline for the management of ingested foreign bodies. Gastrointest Endosc 2002; 55: 802-6.

13. Radford PJ, Wells FC. Perforation of the oesophagus by a swallowed foreign body presenting as a mediastinal and pulmonary mass. Thorax 1988; 43: 416-7.

14. Chung $\mathrm{CH}$. Subtle perforation of the oesophagus by a foreign body. Hong Kong Med J 2003; 9: 290-2.

15. Katsetos MC, Tagbo AC, Lindberg MP, Rosson RS. Esophageal perforation and mediastinitis from fish bone ingestion. South Med J 2003; 96: 516-20.

16. De Lucas EM, Sadaba P, Garcia-Baron PL, et al. Value of helical computed tomography in the management of upper esophageal foreign bodies. Acta Radiol 2004; 45: 369-74.

17. Ng JW. Non-operative management of delayed oesophageal perforation: a note of caution. ANZ J Surg 2005; 75: 1128-30.

18. Metz R, Kimmings AN, Verhagen HJ, et al. Aortoesophageal fistula successfully treated by endovascular stent-graft. Ann Thorac Surg 2006; 82: 1117-9.

19. Tsalis K, Blouhos K, Kapetanos D, et al. Conservative management for an esophageal perforation in a patient presented with delayed diagnosis: a case report review of the literature. Cases J 2009; 2: 6784-4.

20. Sockeel P, Massoure MP, Fixot K, et al. Foreign body perforation of the thoracic esophagus. J Chir 2009; 146: 40-7.

21. Bhat VS, Al-Saadi KA, Bessiouni IE, Tuffaha AS. Embedded esophageal foreign body A diagnostic challenge. Saudi Med J 2009; 30: 433-5.

22. Huang WC, Shen GH, Tseng CW. Persistent right-sided chest pain. Esophageal microperforation by a fish bone associated with empyema thoracis. Gastroenterology 2010; 139: E8-9. 
23. Chen M, Ling Y, Yang BB. Management of late cervical esophageal perforation. Ulus Travma Acil Cerrahi Derg 2010; 16: 511-5.

24. Kerl HU, Zahn K, Kilian AK, et al. A child's birthday with consequences. Radiologe 2010; 50: 1128-31.

25. Chen AP, Yu H, Li HM, et al. Aortoesophageal fistula and aortic pseudoaneurysm induced by swallowed fish bone: a report of two cases. Cardiovasc Interv Radiol 2011; 34: S17-9.

26. Sung SH, Jeon SW, Son HS, et al. Factors predictive of risk for complications in patients with oesophageal foreign bodies. Dig Liver Dis 2011; 43: 632-5.

27. Peng A, Li Y, Xiao Z, Wu W. Study of clinical treatment of esophageal foreign body-induced esophageal perforation with lethal complications. Eur Arch Otorhinolaryngol 2012; 269: 2027-36.

28. Søreide JA, Konradsson A, Sandvik OM, et al. Esophageal perforation: clinical patterns and outcomes from a patient cohort of Western Norway. Dig Surg 2012; 29: 494-502.

29. Ko SF, Lu HI, Ng SH, Kung CT. Fishbone penetration of the thoracic esophagus with prolonged asymptomatic impaction within the aorta. J Vasc Surg 2013; 57: 518-20.

30. Tsukiyama A, Tagami T, Kim S, Yokota H. Use of 3-dimensional computed tomography to detect a barium-masked fish bone causing esophageal perforation. J Nippon Med Sch 2014; 81: 384-7.

Received: 14.07.2017, accepted: 19.10.2017. 